\title{
The STEREO/PLASTIC response to solar wind ions (Flight measurements and models)
}

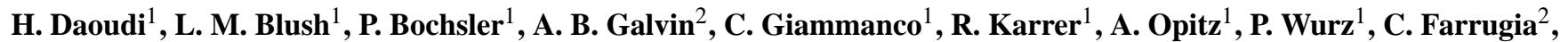 \\ L. A. Kistler ${ }^{2}$, M. A. Popecki ${ }^{2}$, E. Möbius ${ }^{2}$, K. Singer ${ }^{2}$, B. Klecker ${ }^{3}$, R. F. Wimmer-Schweingruber ${ }^{4}$, and B. Thompson ${ }^{5}$ \\ ${ }^{1}$ Physikalisches Institut, University of Bern, 3012 Bern, Switzerland \\ ${ }^{2}$ EOS SSC, University of New Hampshire, Durham, NH 03824, USA \\ ${ }^{3}$ Max-Planck Institut für extraterrestrische Physik, 85740 Garching, Germany \\ ${ }^{4}$ Institute for Experimental and Applied Physics, University of Kiel, 24098 Kiel, Germany \\ ${ }^{5}$ NASA GSFC, Laboratory for Solar and Space Physics, Code 612.1, Solar Physics Branch, Greenbelt, MD 20771, USA
}

Received: 22 November 2007 - Revised: 18 August 2008 - Accepted: 27 November 2008 - Published: 27 March 2009

\begin{abstract}
The Plasma and Supra-Thermal Ion Composition (PLASTIC) instrument is one of four experiment packages on board of the two identical STEREO spacecraft A and $\mathrm{B}$, which were successfully launched from Cape Canaveral on 26 October 2006. During the two years of the nominal STEREO mission, PLASTIC is providing us with the plasma characteristics of protons, alpha particles, and heavy ions. PLASTIC will also provide key diagnostic measurements in the form of the mass and charge state composition of heavy ions. Three measurements $\left(E / q_{k}\right.$, time of flight, $\left.E_{\mathrm{SSD}}\right)$ from the pulse height raw data are used to characterize the solar wind ions from the solar wind sector, and part of the suprathermal particles from the wide-angle partition with respect to mass, atomic number and charge state. In this paper, we present a new method for flight data analysis based on simulations of the PLASTIC response to solar wind ions. We present the response of the entrance system / energy analyzer in an analytical form. Based on stopping power theory, we use an analytical expression for the energy loss of the ions when they pass through a thin carbon foil. This allows us to model analytically the response of the time of flight mass spectrometer to solar wind ions. Thus we present a new version of the analytical response of the solid state detectors to solar wind ions. Various important parameters needed for our models were derived, based on calibration data and on the first flight measurements obtained from STEREO-A. We used information from each measured event that is registered in full resolution in the Pulse Height Analysis words and we derived a new algorithm for the analysis of both existing and future data sets of a similar nature which was tested and works well. This algorithm allows us to obtain, for each
\end{abstract}

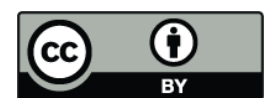

Correspondence to: $\mathrm{H}$. Daoudi (daoudi@space.unibe.ch) measured event, the mass, atomic number and charge state in the correct physical units. Finally, an important criterion was developed for filtering our Fe raw flight data set from the pulse height data without discriminating charge states.

\section{Introduction}

Our Sun is the only star close enough for us to resolve surface details, and also the only star from which matter can be collected. From the upper atmosphere of the Sun, a continuous flow of high-speed particles is ejected from the Sun into the interplanetary medium. In situ measurements of the characteristics of the solar wind ions are possible, and they have previously been carried out by a variety of different spacecraft (Bochsler, 2000). For the first time, 3-D observations of the Sun's corona are now possible at $1 \mathrm{AU}$, thanks to the two identical Solar TErrestrial Relations Observatories (STEREO). One is moving ahead of the Earth in its orbit (STEREO-A) and the other one (STEREO-B) trailing behind. Each spacecraft carries the same four instruments: two for remote imaging investigations (SECCHI and SWAVES) and two for in situ investigations (IMPACT and PLASTIC). The construction of both spacecraft and the integration of all the instruments were accomplished by the Johns Hopkins University Applied Physics Laboratory (APL). The two STEREO spacecraft drift continuously away from Earth at a pace of $22.5^{\circ}$ per year. The estimated nominal duration of the STEREO mission is about two years, during which time the angular distance between the two spacecraft will grow to $90^{\circ}$. The PLAsma and Supra-Thermal Ion Composition sensor (PLASTIC) is designed to measure in situ kinetic properties and charge states of solar wind and suprathermal

Published by Copernicus Publications on behalf of the Arbeitsgemeinschaft Extraterrestrische Forschung e.V. 


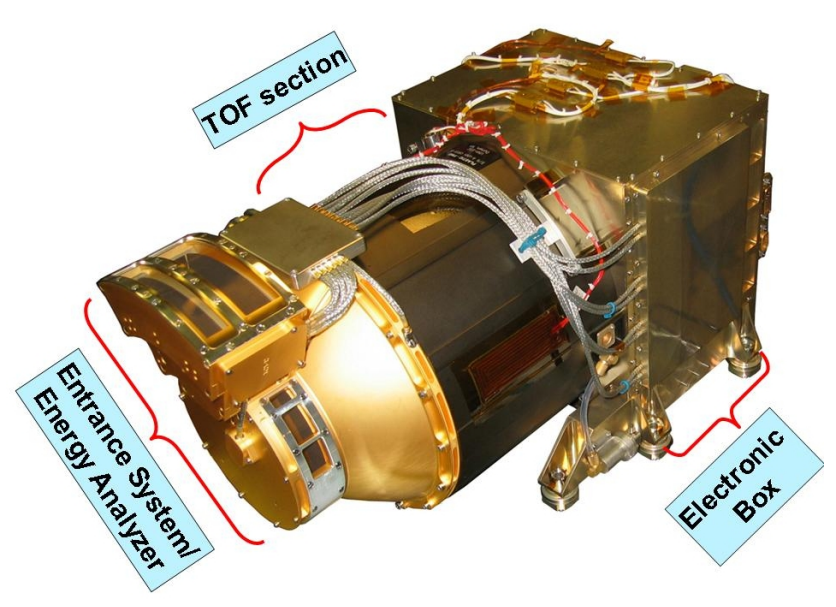

Fig. 1. The PLASTIC instrument with its three different components: Entrance system / energy analyzer (ESEA), the Time Of Flight-Energy section (TOF-E) and the Electronic Box (E-Box).

ions with an energy-per-charge ranging from $0.2 \mathrm{keV} / \mathrm{e}$ to $86.52 \mathrm{keV} / \mathrm{e}$.

In this study, we worked largely with raw-data events. Three measuring concepts are used to characterize the solar wind ions from the Solar Wind Sector (SWS), and a fraction of the suprathermal particles from the Wide-Angle Partition (WAP) with respect to mass $\left(M_{k}\right)$ and charge state $\left(q_{k}\right)$ for a given species $k$. The energy-per-charge $\left(E / q_{k}\right)$ is the first measurement of the PLASTIC sensor. Ions that pass through the Entrance System / Energy Analyzer (ESEA) are first accelerated by a voltage $V_{a}$, so they flow with an energy $E_{\mathrm{tot}}=q_{k} \cdot\left(\frac{E}{q_{k}}+V_{a}\right)$. Then, they pass through the carbon foil where they lose part of their energy $\left(E_{\text {loss,tot }}\right)$. They exit the foil and flow into the Time Of Flight Energy (TOF-E) section with a residual energy $\mathrm{E}_{\text {res }}$. The Time Of Flight (TOF) is the second measurement. The secondary electrons that are emitted from the exit side of the carbon foil are guided toward the Micro Channel Plate (MCP), triggering the start pulse for the TOF. Secondary electrons produced by the ions hitting the Solid State Detector (SSD) are used to trigger the stop pulse for the TOF. Ions that hit the SSDs deposit all their energy in the detector. Only part of this energy can be measured $\left(E_{\mathrm{SSD}}\right) . E_{\mathrm{SSD}}$ represents the third measurement. The main objective of this paper is to present a new algorithm based on a method, which uses every measured event of $E_{\mathrm{SSD}}$ and TOF to derive $M_{k}, Z_{k}$ and $E_{\text {tot }}$ of the solar wind ions. The information of the resulting $M_{k}$ and $E_{\text {tot }}$ is combined with the corresponding measured $E / q_{k}$ at a given $V_{a}$ voltage to determine the charge state $q_{k}$ of a solar wind ion.

In Sect. 2, we give a short description of the PLASTIC instrument. In Sect. 3, the response of the PLASTIC/ESEA for solar wind ions is presented in analytical form. We model the response of the TOF mass spectrometer based on the stopping power theory, valid only for amorphous target materials like our carbon foil. A model of non-channeled mean

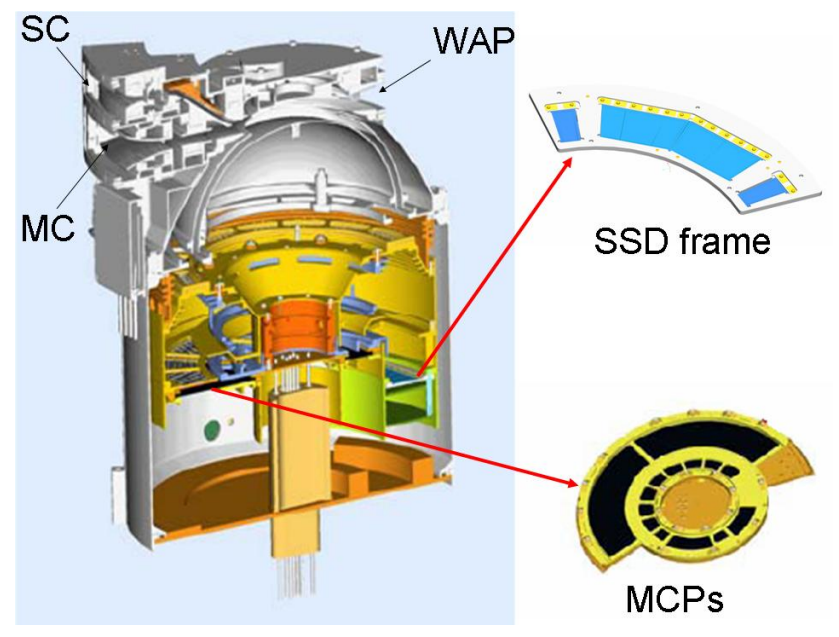

Fig. 2. The image on the left side represents the interior part of the Entrance System / Energy analyzer (ESEA) and the Time Of Flight Energy (TOF-E) sections of the PLASTIC instrument. The picture on the top right side shows a Solid State Detector (SSD) frame with a total of 10 pixels. The picture on the bottom right side shows the Micro-Channel Plate detectors (MCPs).

energy response of the SSDs to solar wind ions is also presented in this chapter. Our PLASTIC/SSD model is valid for all elements and energies up to $2 \mathrm{MeV}$. It is also useful for other crystalline detectors. Still in Sect. 3, we present an algorithm which, from each in situ measurement event, derives the mass, the atomic number and the charge state of solar wind ions with the correct physical units. In Sect. 4, we present a modus operandi for the selection of $\mathrm{Fe}$ data from Pulse Height Analysis (PHA) words.

\section{Description of the PLASTIC instrument}

PLASTIC is designed to measure the in situ kinetic properties of solar wind ions and suprathermal particles. The sensor primarily consists of three different components. An ESEA, which was designed and built by the University of Bern, a TOF-E section and an Electronic Box (E-Box), which were both designed and built by the University of New Hampshire (see Fig. 1).

For the TOF measurements, we use the carbon foil technique, with a foil made by the Max-Planck-Institut für extraterrestrische Physik (MPE) in Germany, with a combination with MCPs detectors from the University of Bern. For the energy measurements we are using SSDs also from the University of Bern, see Fig. 2. All necessary calibrations of the detectors, of the ESEA and of the integrated systems were carried out at the MEFISTO and CASYMS calibration facilities at the University of Bern (Marti et al., 2000; Hohl, 2002). For more information about the design and calibration of PLASTIC, see Blush et al. (2005). 


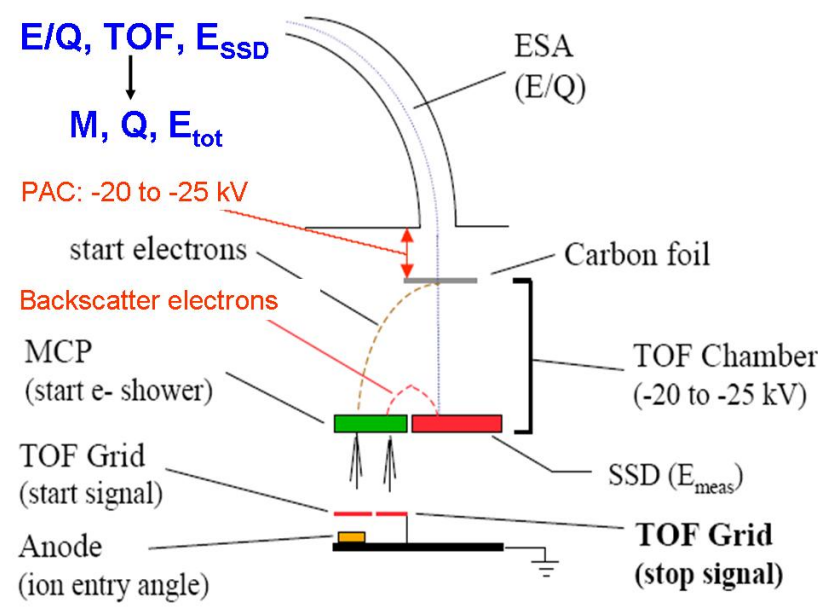

Fig. 3. Schematic view of the PLASTIC SSD-side. The ESEA is followed by the acceleration zone (PAC) and a TOF section. Backward secondary electrons emitted after passing through the carbon foil are detected by the MCP and are used to give the start signal. Forward secondary electrons emitted after the collision with the surface of the SSD are collected in the MCP to give the stop pulse.

\subsection{Entrance System / Energy Analyzer}

Ions of a species k with an initial energy $E$, mass $M_{k}$, and charge state $q_{k}$ enter the ESEA through various apertures designed with different geometric factors.

\subsubsection{Main channel}

The main channel (MC) contains a large geometric factor aperture designed mainly to investigate the distribution functions and charge state distributions for the less abundant solar wind species such as the so called "medium species" $\mathrm{C}, \mathrm{N}$, $\mathrm{O}$, and $\mathrm{Ne}$, and also for the "heavy species" $\mathrm{Mg}, \mathrm{Si}$, Fe etc.

\subsubsection{Small channel}

The small channel (SC) exhibits a smaller geometric factor aperture designed mainly to measure the distribution functions of the abundant solar wind protons and alpha particles. With this aperture; we measure the bulk solar wind plasma parameters (density, velocity, temperature, temperature anisotropy, and alpha/proton ratio). To avoid saturation of the SSDs when a certain count rate is exceeded, the instrument switches automatically to the SC (see Figs. 5a and b). Both, MC and SC, are situated in the SWS.

\subsubsection{Wide angle partition}

The Wide Angle Partition (WAP) is designed especially to measure the distribution functions of suprathermal ions including shock-accelerated particles and pick-up ions. It has a geometric factor similar to the MC and a wide in-ecliptic

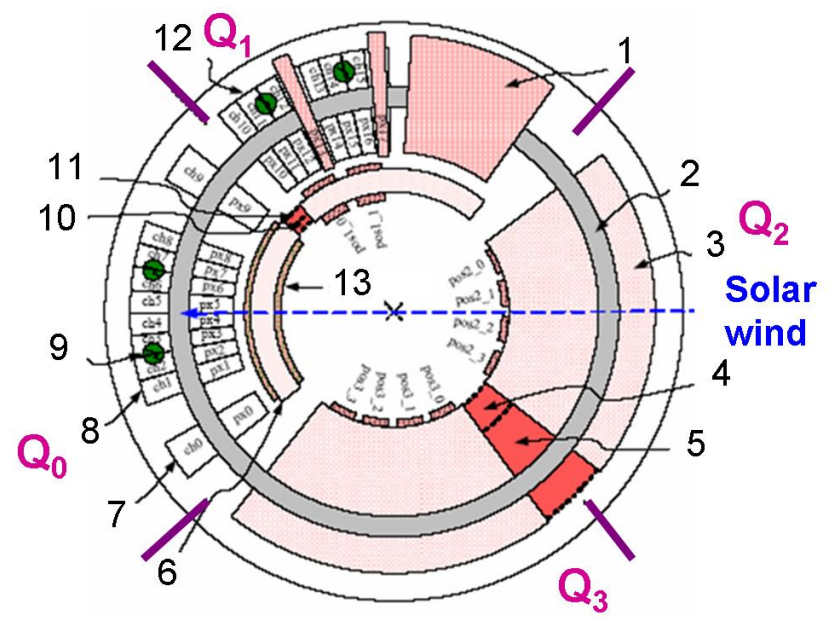

Fig. 4. Schematic top view of the TOF/PLASTIC instrument. Here, the four quadrants can be clearly seen. $\mathrm{Q}_{0}, \mathrm{Q}_{1}$ are on the SSDside and the $\mathrm{Q}_{2}, \mathrm{Q}_{3}$ are on the non SSD-side. Several subsystems necessary for particle detection can also be seen: The white area is covered by SSD pixels [both Solar Wind Sector frame (SWS) and Auxiliary frame (AUX)]. The rose shade corresponds to small and big MCPs. The carbon foils are shown in grey shades. The indices correspond to: 1. Blocked Area (3 places) 2. Carbon-foil (16 segments) 3. Large WAP, MCPs (2 places) 4. Large WAP, Start Grid 5. Large WAP, Stop plate 6. Small MCPs (2 places) 7. WAP SSDs (2 places) 8 . Solar Wind (SW) SSDs (2 each x 4 segments) 9. Thermal sensors (4 places) 10. Small stop plate 11. Small start grid 12. WAP SSDs (2 each x 4 segments) 13. Resistive anode

field of view of $270^{\circ}$ covering the sector that will never be illuminated by the solar wind. This aperture is permanently switched on.

The solar wind ions pass through the ESEA, which works as an energy-per-charge filter for the incoming ions. The energy acceptance range changes during the cycle of operation. It permits the detection of the full solar wind energyper-charge distributions from $0.2 \mathrm{keV} / \mathrm{e}$ to $86.52 \mathrm{keV} / \mathrm{e}$. The energy-per-charge range is scanned in 128 logarithmic steps (see Sect. 3.1 below). The measured $E / q_{k}$ resolution is about $6 \%$. An overview of the instrument science concerning e.g. energy range, resolution, instrument modes, efficiencies etc. is given in several tables in Galvin et al. (2008).

\subsection{Time of flight section}

After leaving the ESEA, the ions enter the acceleration region where they are accelerated by a voltage $V_{a}$, which can be controlled from the ground. (Nominal value $V_{a}=20 \mathrm{kV}$ during the whole mission). The ions then enter the TOF section, first passing through a thin carbon foil with a thickness $d$ of $3.5 \mu \mathrm{g} / \mathrm{cm}^{2}$, where they lose part of their energy $\left(E_{\text {loss,tot }}\right)$, see Eq. (25). Forward secondary electrons are emitted and detected by the MCPs triggering a start time signal (Daoudi, 2008). Ions with a residual en- 


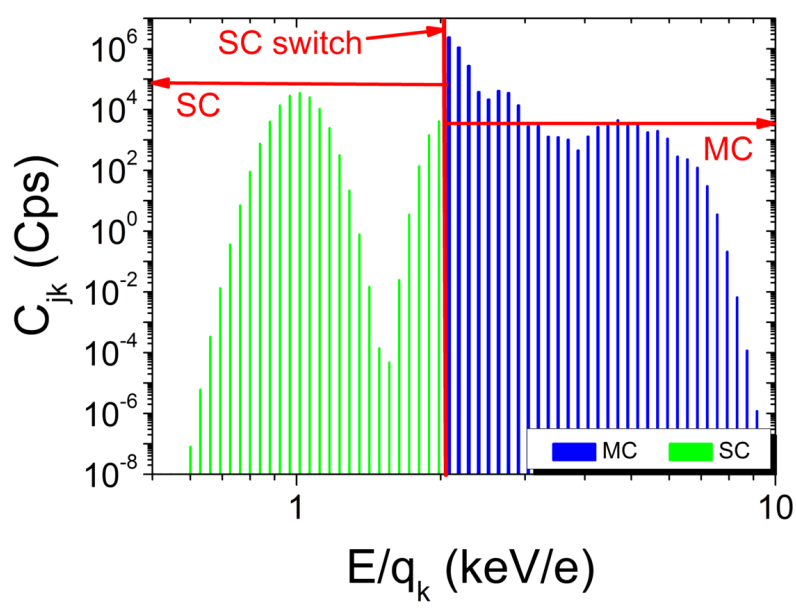

(a)

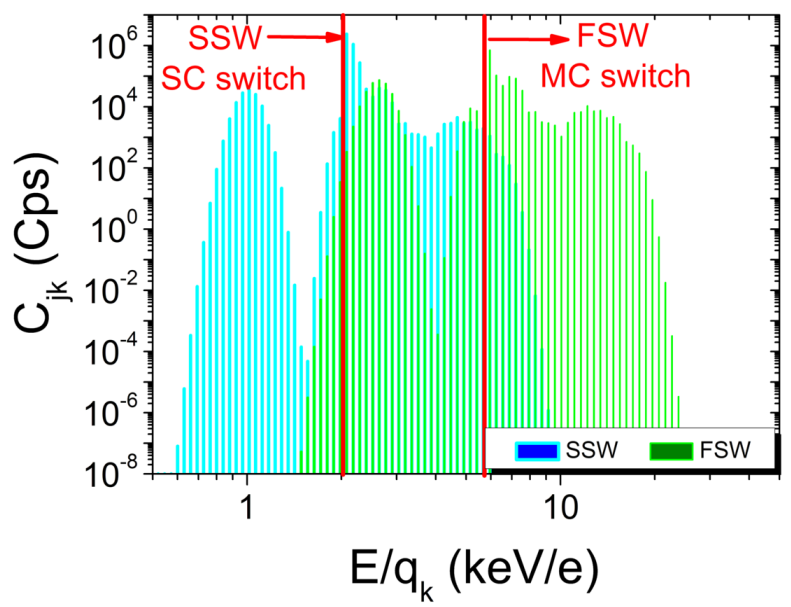

(b)

Fig. 5. (a) Result of the entrance system response simulation to SSW ions. Blue and green shadings represent the simulated count rates measured by the MC and SC respectively. The red line represents the $E / q_{k}$ step from which the DPU sends a command to the instrument to switch on the SC and block the MC. (b) Comparison of the entrance system response to slow and fast solar wind ions (SSW and FSW). We are using the following solar wind parameters for the simulations of the $E / q_{k}$ spectrum in Figs. a and b: (5a) The mean bulk solar wind velocity is $440 \mathrm{~km} / \mathrm{s}$, the mean proton temperature is $2 \cdot 10^{4} \mathrm{~K}$ and the total density is $2 \cdot 10^{6} \mathrm{~m}^{-3}$. (5b) Shows the simulation result from Fig. 5a (cyan shading) compared with the response simulation to fast solar wind ions (olive shading). The two green lines represent the switch to the SC for both SSW and FSW ions regimes. To simulate the FSW we are using a mean bulk solar wind velocity of $720 \mathrm{~km} / \mathrm{s}$, a mean proton temperature of $6 \cdot 10^{4} \mathrm{~K}$ and a total density of $3 \cdot 10^{6} \mathrm{~m}^{-3}$. In a both cases we are assuming that the switch to the SC occurs at count rates $>25 \cdot 10^{5} \mathrm{Cps}$.

ergy $\left(E_{\text {res }}=E-E_{\text {loss,tot }}\right)$ travel a known distance $l$ of $8 \mathrm{~cm}$ through a field-free region (for the SSD-side, see Figs. 3 and 4) and hit the SSD (Quadrants $\mathrm{Q}_{0}$ and $\mathrm{Q}_{1}$ ) losing all of their energy (see Sect. 3.3). In this case backward secondary electrons are emitted from the SSD and detected with one MCP triggering a stop time for the flight signal. If ions travel a distance $l$ of $5.8 \mathrm{~cm}$ (for the non-SSD side, see Fig. 4), they hit another MCP (Quadrants $\mathrm{Q}_{2}$ and $\mathrm{Q}_{3}$ ), where the ion generates an electron cascade that turns into a detectable charge signal, triggering a stop signal. The difference between stop and start times gives the corresponding TOF.

\subsection{Electronic box}

The electronic box produces the electronics commands for the operation of the instrument and all the subsystems for data processing. It includes also power supplies, which provide voltages to the instrument components (electrode, bias, $V_{a}$ voltage... ). For more details, consult Galvin et al. (2008).

\section{Modeling the PLASTIC instrument response}

PLASTIC provides raw event data (PHA) which are used for high-resolution science, as well as instrument diagnostics in the form of counts at a given energy-per-charge step $(j)$, in-ecliptic angle $(\alpha)$, out-of-ecliptic angle $(\beta)$, TOF, $E_{\mathrm{SSD}}$ etc. This information can be used to derive the mass and the charge state of the solar wind ions, but the results must be converted into correct physical units by considering the energy defect in the SSD measurements and the energy lost in the carbon foil.

In order to calculate these values, we created an appropriate algorithm that takes into account all the information from our simulations of the instrument's response to solar wind ions and uses the raw event data $\left(j, T O F, E_{\mathrm{SSD}}\right)$ from the PHA files as input parameters. Our algorithm calculates the corresponding masses and charge states of all the ions in the correct physical units without requiring separate treatment of different elements. The simulation response is based on stopping power theory and on the calibration results of our instruments and detectors. In the next section, we compare the ESEA response to slow and fast solar wind ions (SSW and FSW).

\subsection{Simulation of the ESEA response}

In Figs. 5a and $\mathrm{b}$ we simulate an energy-per-charge spectrum of solar wind ions which have passed the ESEA through both, $\mathrm{MC}$ and SC apertures. We define the energy-per-charge $E / q_{k}$ in every step $j$ according to the expression:

$$
\left(\frac{E}{q_{k}}\right)_{j}=D_{1} \cdot D_{2}^{(127-j)} ; \quad j=0, \ldots \ldots, 127
$$

where $D_{1}=0.2 \mathrm{keV} / \mathrm{e}$ represents the lowest energyper-charge value, the relative increase is given by 
$D_{2}=1.048954356$ and the value of 127 is the maximum step number used.

At a given step $j$, an ion $k$ with a mass $M_{k}$, and a charge state $q_{k}$ passes through the entrance system with an acceptance speed $v_{j k}$, which is defined as:

$v_{j k}=\sqrt{\frac{2 q_{j k}}{M_{j k}} \cdot\left(\frac{E}{q_{k}}\right)_{j}}$

The coordinate system used is centred on the spacecraft, with the $x$ axis pointing anti-sunward, the $y$ axis lying in the ecliptic plane and pointing in the direction of the Earth's motion; and the $z$ axis completing the right-handed orthogonal coordinate system. The count rate, $C_{j k}$, for a particle $k$ passing through the entrance system at a given step $j$ was calculated for earlier experiments (e.g. Oetliker, 1993) to be the integral of the flux of particles through the Active Area $\boldsymbol{A}$ of the instrument (in our example the entrance apertures of $\mathrm{MC}$ or SC respectively), and the distribution function $f(\boldsymbol{v})$ of the plasma to be analyzed:

$C_{j k}=\int \boldsymbol{A} \cdot \boldsymbol{v} f(\boldsymbol{v}) d^{3} v$

For the active area, we use the procedure of Aellig (1998) and Hefti (1997) which is based on the ion optical calibration and the geometry of the entrance system. We employ a dimensionless transmission probability $t(\alpha, \beta, v, j)$ in step $j$, which depends on the entrance angles into the instrument ( $\alpha$ and $\beta$ ), and the speed of the incoming particles. So Eq. (3) can be written as follows:

$C_{j k}=\int A t(\alpha, \beta, v, j) v f(\boldsymbol{v}) d^{3} v$

We separate the transmission function of the instrument into three different acceptance functions $t(\alpha, \beta, v, j)=t_{1}(\alpha) t_{2}(\beta) t_{3}(v, j)$, assuming that the dependences on the parameters $\alpha, \beta$ and $v$ do not influence each other.

The measured $\alpha$ acceptance is approximated by a rectangle of half $\alpha_{c}=22^{\circ}$ width, for the measured $\beta$ transmission function we define a cutoff value $\beta_{c}$ which describes an interval $\left[-\beta_{c}, \beta_{c}\right]$ over which the transmission $t_{2}(\beta)$ is fitted with a Gaussian function with a standard deviation of $\sigma_{\beta}=1.8^{\circ} / 2 \sqrt{\ln 4}$ measured for the MC and $\sigma_{\beta}=0.3^{\circ} / 2 \sqrt{\ln 4}$ measured for the SC. The measured velocity transmission function for nominal entrance $(\alpha=\beta=0)$ is also fitted using a Gaussian function.

We represent the velocity transmission function at a given step $j$ by:

$t_{3}(v, j)=\exp \left[-\frac{\left(v-v_{j}\right)^{2}}{2\left(\varepsilon_{v} v_{j}\right)^{2}}\right]$

where $\varepsilon_{v}=0.03 / 2 \sqrt{\ln 4}$ represents the relative width of the velocity transmission function. For the active area and the geometric factor, values in Table 1 are used.
Table 1. FM1 active area and geometric factor for both MC and SC apertures.

\begin{tabular}{|c|c|c|}
\hline FM1 & $\begin{array}{c}\text { Active Area } \\
\text { (in } \mathbf{~ c m}^{2} \text { ) }\end{array}$ & $\begin{array}{c}\text { Geometric Factor } \\
\text { (in } \mathbf{~ c m}^{2} \mathbf{~} \mathbf{V} / \mathbf{e V} \text { sr per } \mathbf{4 5}^{\circ} \text { sector } \text { ) }\end{array}$ \\
\hline MC & $8.910^{-1}$ & $3.1510^{-3}$ \\
\hline SC & $1.4110^{-3}$ & $6.010^{-7}$ \\
\hline
\end{tabular}

For more details about the ion optical calibrations of the ESEA see Karrer (2007). We write $C_{j k}$ as follows:

$C_{j k}=\int A t_{1}(\alpha) t_{2}(\beta) t_{3}(v, j) v f(\boldsymbol{v}) d^{3} v$

As a simplification, we assume that solar wind ions are drifting in a three dimensional spherical Maxwellian distribution with a bulk drift speed $v_{0 k}\left(v_{0}, 0,0\right)$ with respect to our coordinate system. For the following calculations, we will transform the Cartesian coordinates into spherical coordinates. Using the definition of the volume $V$ in velocity space we can write $d^{3} v$ as a function of the differentials of $v, \alpha$ and $\beta$ as follows:

$$
\begin{aligned}
d^{3} v=d V & =\left|\frac{\partial\left(v_{x}, v_{y}, v_{z}\right)}{\partial(v, \alpha, \beta)}\right| d v d \alpha d \beta \\
& =v^{2} \cos \beta d v d \alpha d \beta
\end{aligned}
$$

Using Eq. (7), the count rate can be written in the following form:

$C_{j k}=\iiint A v^{3} \cos \beta t_{1}(\alpha) t_{2}(\beta) t_{3}(v, j) f(\boldsymbol{v}) d v d \alpha d \beta$

In this example, we are assuming that the temperatures parallel and perpendicular to the magnetic field are equal. The solar wind distribution is given by:

$$
\begin{aligned}
& f\left(v_{x}, v_{y}, v_{z}\right)=n_{k}\left(\frac{M_{k}}{2 \pi k_{B} T_{k}}\right)^{3 / 2} \\
& \exp \left[-\frac{M_{k}}{2 k_{B} T_{k}}\left(\left(v_{x}-v_{0}\right)^{2}+v_{y}^{2}+v_{z}^{2}\right)\right]
\end{aligned}
$$

where $T_{k}$ is the temperature and $n_{k}$ the density of the species $k$. Quantity $k_{B}$ is the Boltzmann constant . Now if we transform $v_{x}, v_{y}$ and $v_{z}$ into spherical coordinates, we expand all trigonometrically terms of $\alpha$ and $\beta$ from the resulting substitution of the spherical coordinates in the Eq. (9) until the second order, and we change the variable $v$ into a new variable $\delta$ defined in such a manner that when $\delta$ tends to zero, then $v$ will tend to the speed $v_{0}$, and we do this by defining $v=(1+\delta) v_{0}$. Then, we find that:

$$
\begin{aligned}
& \left(v_{x}-v_{0}\right)^{2}=v_{0}^{2} \delta^{2} \\
& v_{y}^{2}=v_{0}^{2} \alpha^{2} \\
& v_{z}^{2}=v_{0}^{2} \beta^{2}
\end{aligned}
$$




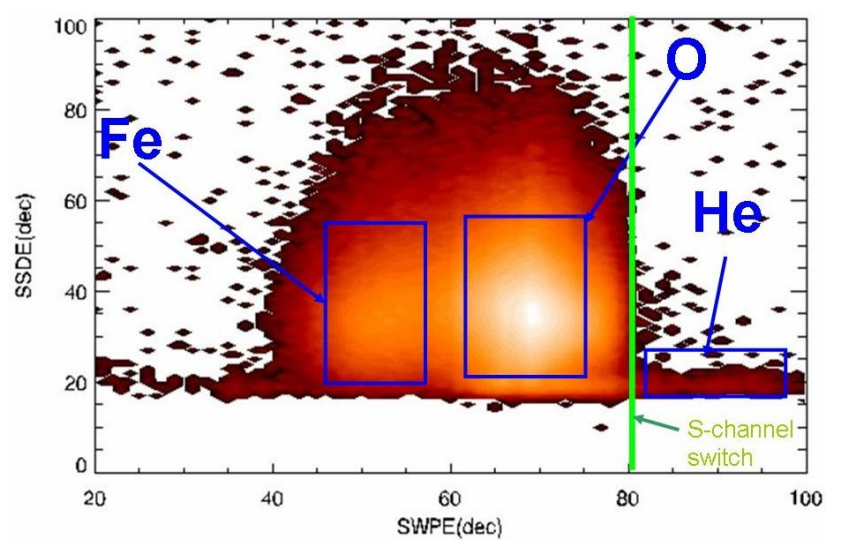

Fig. 6. Raw data of the energy measured by the PLASTIC instrument on board STEREO-A versus the sweeping step number $\mathrm{j}$ (SWPE). For an accumulation time of $24 \mathrm{~h}$ on 22 January 2007. Higher $\mathrm{j}$ values correspond to lower energy-per-charge $\left(E / q_{k}\right)$. He, $\mathrm{O}$ and $\mathrm{Fe}$ can be distinguished.

We substitute the transmittance functions, we change the variable $v$ into $\delta$, we expand the term $(1+\delta)^{3}$ up to second order, and we make all the necessary substitutions in Eq. (8). The resulting count rate integral is:

$C_{j k}=n_{k}\left(\frac{1}{2 \pi k_{B} T_{p k}}\right)^{3 / 2} A I I_{\alpha} I I_{\beta} I I_{\delta}$

where :

$$
\begin{aligned}
I I_{\alpha}= & \int_{-\alpha_{c}}^{+\alpha_{c}} \exp \left(-\frac{v_{0}^{2}}{2 k_{B} T_{p k}} \alpha^{2}\right) d \alpha \\
I I_{\beta}= & \int_{-\beta_{c}}^{+\beta_{c}} \exp \left(-\left(\frac{v_{0}^{2}}{2 k_{B} T_{p k}}+\frac{1}{2 \sigma_{\beta}^{2}}\right) \beta^{2}\right) d \beta \\
& \int_{\delta}^{+\infty} v_{0}^{4}(1+\delta)^{3} t_{3}(\delta, j) \exp \left(-\frac{v_{0}^{2}}{2 k_{B} T_{p k}} \delta^{2}\right) d \delta
\end{aligned}
$$

After many calculations, and simplifications we solved the three integrals (14), (15) and (16). For more details concerning the solution of this integrals, see Daoudi (2008). As a result, the following equation shows the analytical expression of the count number of the species $k$ per unit time that passes the ESEA at a given step $j$ :

$C_{j k}=f_{1} \cdot\left[f_{2}+f_{3}\right]$

For simplicfication, three functions were used in the expres- sion for $C_{j k}$. They are defined as follows:

$$
\begin{aligned}
& f_{1}=n_{k} A v_{0} \cdot\left(\sqrt{1+\frac{k_{B} T_{p k}}{v_{0}^{2} \sigma_{\beta}^{2}}}\left(1+\frac{k_{B} T_{p k}}{\varepsilon_{v}^{2} v_{j k}^{2}}\right)^{\frac{3}{2}}\right)^{-1} \\
& {\left[2 \phi\left(\alpha_{c} \sqrt{\frac{v_{0}^{2}}{k_{B} T_{p k}}}\right)-1\right]} \\
& {\left[2 \phi\left(\beta_{c} \sqrt{\frac{v_{0}^{2}}{k_{B} T_{p k}}+\sigma_{\beta}^{-2}}\right)-1\right]} \\
& f_{2}=\frac{3 k_{B} T_{p k}}{v_{0}^{2}} \exp \left(-\frac{\left(v_{0}-v_{j k}\right)^{2}}{2 \varepsilon_{v}^{2} v_{j k}^{2}}\right) \\
& f_{3}=\exp \left(-\frac{\left(v_{0}-v_{j k}\right)^{2}}{2\left(k_{B} T_{p k}+\varepsilon_{v}^{2} v_{j k}^{2}\right)}\right) \\
& {\left[1+\frac{k_{B} T_{p k}}{\varepsilon_{v}^{2} v_{j k}^{2}}\left(3 \frac{v_{j k}}{v_{0}}-2\right)\right.} \\
& \left.+\frac{3\left(v_{0}-v_{j k}\right)^{2}\left(\frac{k_{B} T_{p k}}{\varepsilon_{v}^{2} v_{j k}^{2}}\right)^{2}}{v_{0}^{2}\left(1+\frac{k_{B} T_{p k}}{\varepsilon_{v}^{2} v_{j k}^{2}}\right)}\right]
\end{aligned}
$$

with $T_{p k}$ denoting the temperature per mass. $\phi(y)$ is the cumulative area under a standard normal distribution from minus infinity to a particular point " $y$ " along the curve, and it is defined to be:

$\phi(y)=(2 \pi)^{-\frac{1}{2}} \int_{-\infty}^{y} e^{-\frac{x^{2}}{2}} d x$

Figures $5 \mathrm{a}$ and $\mathrm{b}$ show the results of the simulation of the energy-per-charge spectrum for both FSW and SSW ions.

Figure 6 shows the raw event data in energy and energy-per-charge from the PHA flight data measured with STEREO-A. Increasing sweep steps produce lower $E / q_{k}$ selection. The information about the $E / q_{k}$ ratio, combined with the TOF and $E_{\mathrm{SSD}}$ measurements from each event, will be used as input for our algorithm in order to calculate the mass and charge state of the incident solar wind ions.

On 22 January 2007 the solar wind had the same parameters as those used in the simulations shown in Fig. 5a. The data represent an accumulation of counts from both MC and $\mathrm{SC}$, and from all quadrants and all SSD pixels. We can see that the energy plot is dominated by oxygen and iron since they were detected through the MC aperture, while helium ions were mainly detected through the SC. Protons are below the threshold of the SSD in this plot. 


\subsection{Time of flight simulation response}

In order to perform simulations of the TOF spectrum we need to evaluate the energy lost by these ions when they pass through the thin carbon foil (amorphous material), and compute the final distribution of these ions. We then derive an analytical expression for the TOF as a function of both the nuclear and the electronic energy loss, of the ions.

When energetic ions hit matter, they lose kinetic energy. For our case, we do not consider nuclear reactions or the effect of "bremsstrahlung", since these are significant only at higher energies. We assume that the stopping power is a simple superposition of the nuclear and electronic stopping powers. Nuclear stopping refers to the energy transferred from the ions to the atoms of the target and occurs in elastic interatomic collisions. At low speeds, this process dominates the energy transfer; the ions lose most of their energy in collision cascades and their direction can be changed considerably. At higher energies, the major part of the energy of the projectile ions will be transferred to the electrons of the target material (electronic stopping) see Fig. 7.

Generally, for a given target, the behaviour of the stopping power is the same for all ions, with only small differences in the offset of the $\log \left(S_{e}\right)$ curve, or of the maximum point of the $\log \left(S_{n}\right)$ curve. The velocity at which the nuclear stopping reaches a maximum is approximately the same for all ions, and mainly depends on the target material.

We used the ZBL universal screening length (Ziegler et al., 1992), which is also used by Crystal TRIM (CTRIM), with some modifications (Pilz et al., 1998). We calculated the nuclear energy lost by the ions passing through a target with a thickness $d[\mathrm{~nm}]$; this yielded a result similar to the Betz and Wien (1994) formula but with a different screening length:

$$
E_{\mathrm{loss}, n}=d \frac{509.59 \rho Z_{k} Z_{2} M_{k}}{M_{2}\left(M_{k}+M_{2}\right)\left(Z_{k}^{0.23}+Z_{2}^{0.23}\right)} S_{n}(\varepsilon) \quad[\mathrm{eV}]
$$

with the dimensionless nuclear stopping power defined as:

$$
S_{n}(\varepsilon)= \begin{cases}\frac{\ln (1+1.1386 \varepsilon)}{2\left(\varepsilon+0.01321 \varepsilon^{0.21226}+0.19593 \varepsilon^{0.5}\right)} \\ \text { if } \varepsilon \leq 30 \\ \frac{\ln (\varepsilon)}{2 \varepsilon} & \text { if } \varepsilon>30\end{cases}
$$

where $\rho\left[\mathrm{g} / \mathrm{cm}^{3}\right]$ is the carbon foil density and $\varepsilon$ is the corresponding Firsov reduced energy, which is defined as follows:

$\varepsilon=\frac{32.53 M_{2} E_{\mathrm{tot}}}{Z_{k} Z_{2}\left(M_{k}+M_{2}\right)\left(Z_{k}^{0.23}+Z_{2}^{0.23}\right)}$

Here, $M_{k}$ [amu], $M_{2}$ [amu], $Z_{k}$, and $Z_{2}$ are the mass and the atomic number of the projectile (index $\mathrm{k}$ ) and the target element (index 2), respectively. $E_{\text {tot }}[\mathrm{keV}]$ is the total initial energy of the ions before passing through the carbon foil.

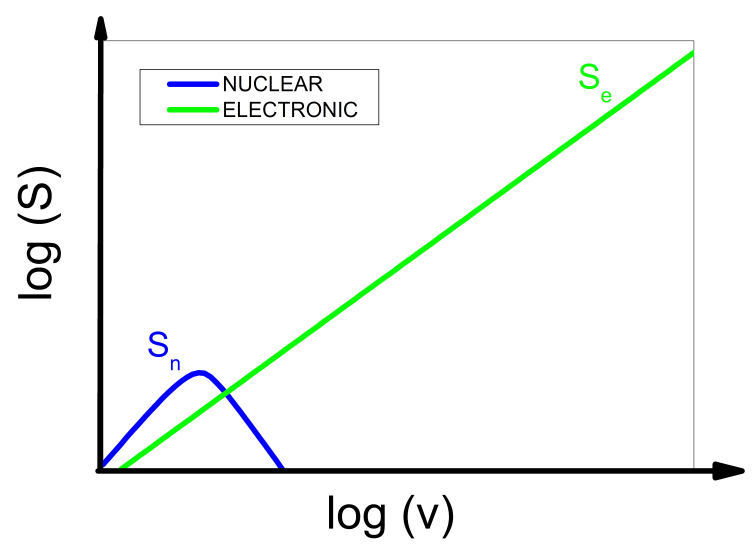

Fig. 7. Schematic plot of the behaviour of the nuclear and electronic energy losses per unit path length (respectively $S_{n}$ and $S_{e}$ ) as functions of the velocity of the projectile in cases where Firsov reduced energies $\varepsilon \leq 30$

To calculate the energy lost due to electronic stopping we use the expression (24) from Betz and Wien (1994).

$E_{\text {loss }, e}=d \frac{231.6 \rho Z_{\mathrm{keff}}^{7 / 6} Z_{2}}{\left(Z_{\mathrm{keff}}^{2 / 3}+Z_{2}^{2 / 3}\right)^{3 / 2} M_{2} \sqrt{M_{k}}} \sqrt{E_{\mathrm{tot}}} \quad[\mathrm{eV}]$

The total energy lost after the carbon foil is then calculated as a simple addition of both, nuclear and electronic energy loss:

$E_{\mathrm{loss}, \mathrm{tot}}=E_{\mathrm{loss}, n}+E_{\mathrm{loss}, e}$

$Z_{\text {keff }}$ represents the effective charge defined as a function of the ion velocity $(v[\mathrm{~m} / \mathrm{s}])$, the atomic number of the projectile $\left(Z_{k}\right)$ and the Bohr velocity $\left(v_{0}[\mathrm{~m} / \mathrm{s}]\right)$ as follows:

$Z_{\mathrm{keff}}=Z_{k}\left[1-\exp \left(-Z_{k}^{-2 / 3} \frac{v}{v_{0}}\right)\right]$

The residual energy of the ions after passing through the carbon foil will be:

$E_{\text {res }}=q_{k}\left(\frac{E}{q_{k}}+V_{a}\right)-E_{\text {loss,tot }}$

Finally the simulated TOF can be calculated to be:

$\mathrm{TOF}=l \sqrt{\frac{M_{k}}{2 E_{\mathrm{res}}}}$

Figure $8 \mathrm{a}$ shows the simulation result of the nuclear, electronic, and total energy loss of helium ions in our thin carbon foil, calculated using the Eqs. (21), (24) and (25) respectively as functions of the projectile energy.

We performed measurements with the integrated system flight model 1 (IFM1), for various species and different energies in the facilities of the University of Bern. The TOF 

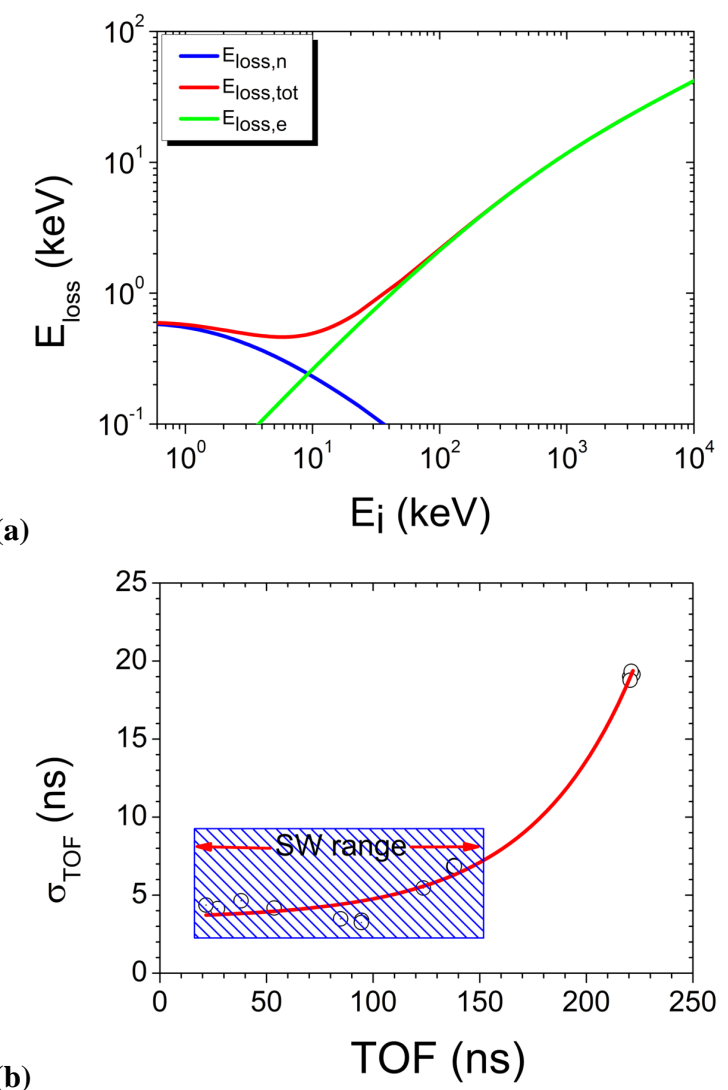

(b)

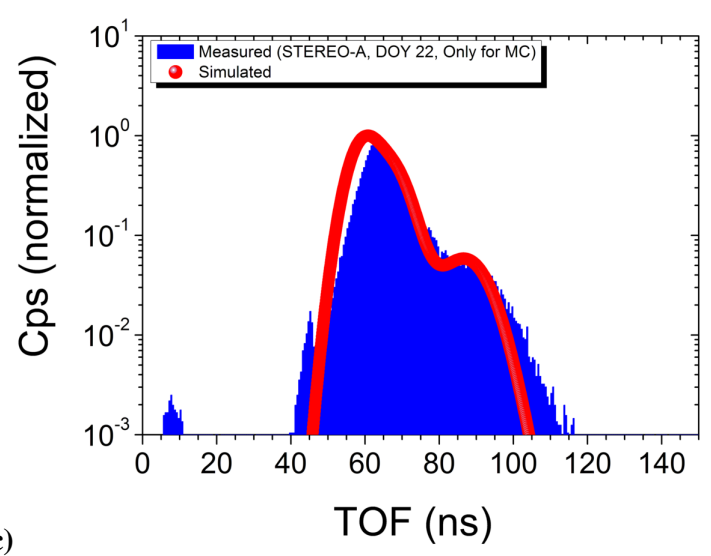

Fig. 8. (a) Simulated energy loss $\left(E_{\text {loss }}\right)$, of helium in a thin carbon foil $\left(3.5 \mu \mathrm{g} / \mathrm{cm}^{2}\right)$ as a function of the projectile energy $\left(E_{i}\right)$. The blue, green and red curves are the estimated nuclear, electronic and total energy loss respectively. (b) Standard deviation of the TOF gauss fit spectra for various species $\mathrm{H}, \mathrm{He}, \mathrm{O}$ and $\mathrm{Ar}$ and diverse energies (black data points). The blue quadrant represents the TOF range of solar wind ions, and the red curve is the exponential fit to our data. (c) Comparison of the shape of the measured and simulated TOF spectra to SSW ions entering the MC aperture, with a mean bulk velocity of $440 \mathrm{~km} / \mathrm{s}$. We are using the same solar wind parameters as those used in Figs. 6 and 5a. For the simulated spectrum (red curve) we are using an acceleration voltage $V_{a}$ of $17.3 \mathrm{kV}$, and we are assuming that the switch to the SC occurred at count rates $>25 \cdot 10^{5} \mathrm{Cps}$. Both spectra are normalized. histograms from the calibration results were fitted by a Gaussian function, the corresponding standard deviation is shown in the Fig. $8 \mathrm{~b}$.

Figure 8c shows the comparison of the shape of the TOF spectra simulated and measured with STEREO-A. The calculation is performed using the Eq. (17) and the resolution of the TOF spectra from Fig. 8b. We are using the same solar wind conditions as those in Fig. 5a (accumulation time of $24 \mathrm{~h}$ on the 22 January 2007). Both measurements and simulations are only for the MC aperture. Some discrepancies stem from the measured background data; they might be due mainly to solar UV photons and to some differences in the switch to the SC count rate. For more details, see Daoudi (2008).

\subsection{Modeling of the response of PLASTIC solid state detectors to light, medium, and heavy species}

Various branches of physics exploit SSDs for charged particle spectroscopy. Generally, SSDs use semiconductor materials such as Germanium, Silicon (in crystalline form), Cadmium Zinc Telluride (CdZnTe), etc. They offer better resolution, lower noise, and better spatial resolution than the ordinary scintillator detectors, which used "activator" materials such as Thallium and Sodium.

The PLASTIC/SSDs employed are Passivated Implanted Planar Silicon (PIPS) detectors. They were developed, designed, and manufactured by CANBERRA industries. When a charged particle penetrates such a detector, its entire energy is deposited mainly via two mechanisms: electron and nuclear interactions. Neither the energy transmitted to the nuclei (phonons) nor the energy lost in the Dead Layer (DL) is detected. However, the energy needed to create electronhole pairs $\left(\mathrm{e}^{-} / \mathrm{p}\right)$ in silicon is a constant of $3.6 \mathrm{eV}$. The energy transferred to the electrons of the target semiconductor from the incident ions that will generate $\left(\mathrm{e}^{-} / \mathrm{p}\right)$ is then drained by the electric field across the semiconductor, and this creates an electric current that can be detected, amplified, and measured. $\left(\mathrm{e}^{-/ \mathrm{p}}\right)$ pairs are created continuously until the ions lose all of their initial energy and are stopped in the detector. The theoretical treatment of the stopping power of an ion in matter is best known when the tagret material is amorphous (Ziegler et al., 1992; Biersack, 1982), as we have seen in the Subsect. 3.2 when our material was a thin carbon foil. In such amorphous materials, there is no long-range order of the positions of the atoms, and the stopping power of the ions can be predicted accurately in an analytical form using the transport theory or Monte Carlo simulations (Ziegler et al., 1992). But when the atoms or ions of the target material are arranged in an orderly manner as in our PLASTIC/SSDs or other crystalline detectors, then the same lattice performs differently depending on the viewing aspect. Consequently, the stopping power depends strongly on the incident angles of the ions with respect to the crystal lattice. Anomalies of the detector response (channeling effect) can occur and the 


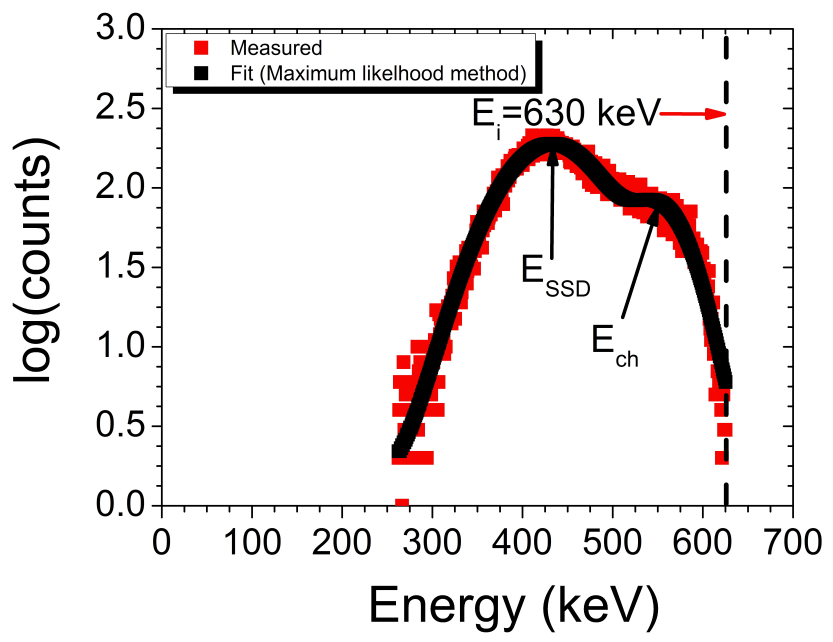

Fig. 9. Pulse Height Energy Analyzer Spectrum (PHEAS). The red data points are the measurements and the black curve is a fit function to the Ar data using a sum of two Gaussian distributions.

measured energy distribution will be far from having a Gaussian shape. This is the cause that the stopping power in such a crystalline structures is not very well known. For this reason, to model our SSD response to solar wind ions, we use a treatment that is different from the one with the carbon foil. Many attempts to simulate the response of silicon detectors have been made (Pilz et al., 1998) in the past. Most of them used Monte Carlo simulations, Binary Collision Approximation (BCA) or Molecular Dynamics (MD) methods, but these do not result in a conclusive model. The response of the SSDs depends strongly on the orientation of the silicon wafer crystal, the DL of the target material, as well as the incident ion's properties, such as the angle with respect to the crystal lattice, its energy and its mass. Since, we need to reconstruct the initial energy for all species before they hit the detector, we have studied the behaviour of the initial incident energy before the ions hit the $\operatorname{SSD}\left(E_{i}\right)$ as a function of the mean measured energy $\left(E_{\mathrm{SSD}}\right)$ for various elements.

\subsubsection{PLASTIC/SSD characteristics}

PLASTIC SSDs are mounted in Macor frames. One of them, the Solar Wind (SW) frame, contains a total of ten SSD trapezoid pixel shapes. It is located in Quadrant $\mathrm{Q}_{0}$ in the SWS. The other one is the auxiliary (AUX) frame, which contains a total of eight SSD pixels. It is placed in $\mathrm{Q}_{1}$ in the WAP sector (see Fig. 4).

Each pixel of PLASTIC/SSDs is 502 microns thick with an effective active area of $95 \mathrm{~mm}^{2}$, and a thin silicon entrance window of $25 \mathrm{~nm}$. The operating voltage in the instrument is $95 \mathrm{~V}$. The gamma resolution FWHM is $6.6 \mathrm{keV}$ as measured with an ${ }^{241} \mathrm{Am}$ source. SSD detectors are stable, with a low leakage current and low noise. The measured threshold of our detector is about $25 \mathrm{keV}$. The lower the threshold,

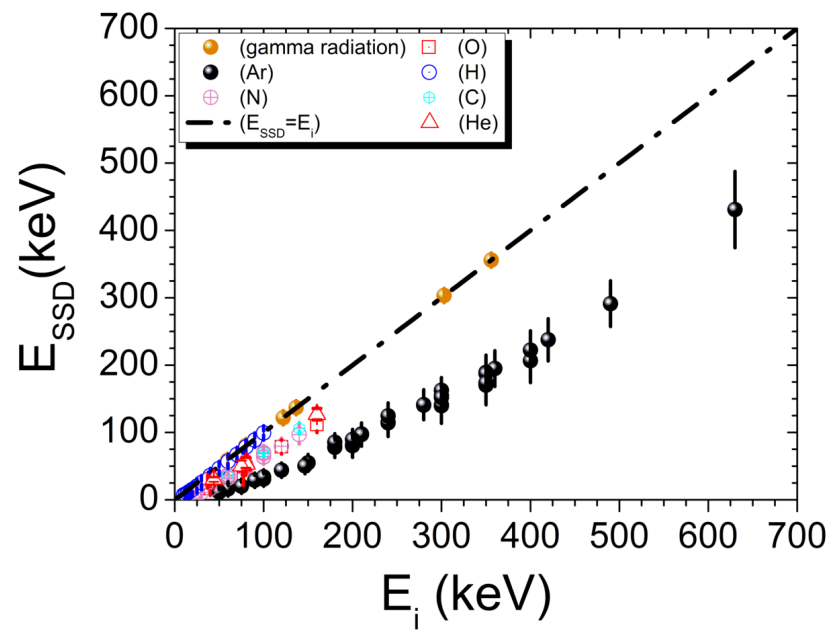

Fig. 10. SSD mean measured energy for non-channeled ions as a function of the projectile energy $E_{i}$ (direct irradiation of the beam into the SSD pixels for gamma radiation, $\mathrm{H}, \mathrm{C}, \mathrm{N}, \mathrm{O}$ and $\mathrm{Ar}$ ). In the case of He we use measurements from the calibrations of the integrated FM1 (indirect irradiation into the SSD). The error bars are the measured FWHM.

the greater the probability of detecting, low-energy, singly charged ions.

\subsubsection{Experimental data and model}

To start with, calibration measurements were made for the SSDs in the MEFISTO facility at the University of Bern using gamma sources such as ${ }^{57} \mathrm{Co},{ }^{133} \mathrm{Ba}$, and ${ }^{241} \mathrm{Am}$. Next, the energy response was measured for different ions $\left(\mathrm{H}^{+}\right.$, $\mathrm{C}^{2+}, \mathrm{N}^{2+}, \mathrm{O}^{2+}, \mathrm{Ar}^{5+}, \mathrm{Ar}^{6+}$ and $\mathrm{Ar}^{7+}$ ) and at energies from $9 \mathrm{keV}$ to $630 \mathrm{keV}$. Figure 9 shows a Pulse Height Energy Analyzer Spectrum (PHEAS) for $\mathrm{Ar}^{7+}$ at $90 \mathrm{keV} / \mathrm{e}$.

Silicon in crystalline form has five major channeling cen-

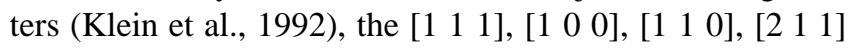
and $\left[\begin{array}{lll}4 & 1 & 1\end{array}\right]$ directions. Channeling occurs when the incident ion can move long distances without nuclear collision compared to other directions. Consequently, the amount of energy lost by nuclear scattering of the ion in a detector decreases. The electron density in a channel is also usually lower than the average density, hence, these two effects lead to a lower stopping power. Each PLASTIC/SSD pixel is cut from a silicon wafer at an angle of $7^{\circ}$ off the [lllll 111$]$ axis to minimize the channeling effect. Still, we found that it was not good enough to prevent channeling effect for heavy ions. Consequently, the measured pulse height distribution was broader, and the energy resolution decreased. Since we measured energy from both channeled and non-channeled ions, all energy spectrum calibrations of all the SSD pixels and of both integrated systems FM1 and FM2 (IFM1 and IFM2) were fitted with a sum of two Gaussian distributions; the first Gaussian corresponds to the measured energy $E_{\mathrm{SSD}}$ 


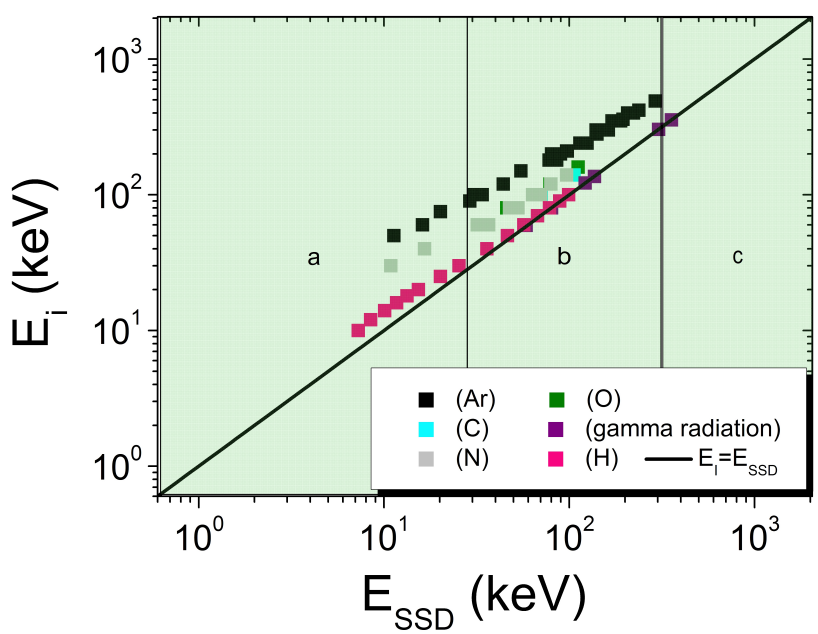

Fig. 11. Same as in Fig. 10, but in logarithmic scale (for gamma radiation, $\mathrm{H}, \mathrm{C}, \mathrm{N}, \mathrm{O}$ and $\mathrm{Ar}$ ).

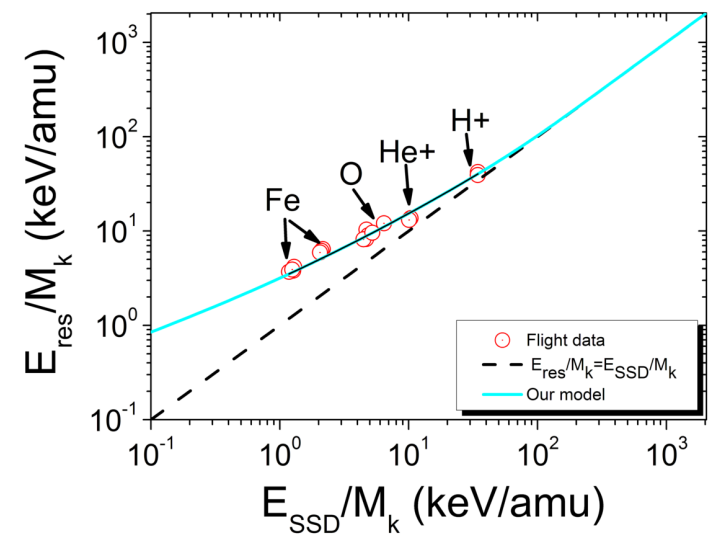

Fig. 12. The red data points mark the residual energy of different ions before hitting the SSD versus the mean energy measured with the SSDs. The data are from STEREO-A/PLASTIC for different days between 22 January and 11 February 2007. The cyan line represents our model for energy ranges up to $200 \mathrm{keV} / \mathrm{amu}$. The red curve represents the fit to the data with our model.

(non-channeled ions or randomly scattered ions), the second $\left(E_{c h}\right)$ corresponds to channeld ions (see Fig. 9).

Figures 10 and 11 show the mean measured energy as a function of the incident energy for different species. The behaviour seems to be similar for all species.

Our idea was to build a numerical model using an analytical function which must be able to describe three different types of behaviours in three different zones, $\mathbf{a}, \mathbf{b}$ and $\mathbf{c}$ (see Fig. 11):

Zone a: The mean energy measured for all the ions at very low energies must tend to zero.

Zone b: In the intermediate region, the model must follow a power law.

Zone c: For higher energies, the curve will approach the

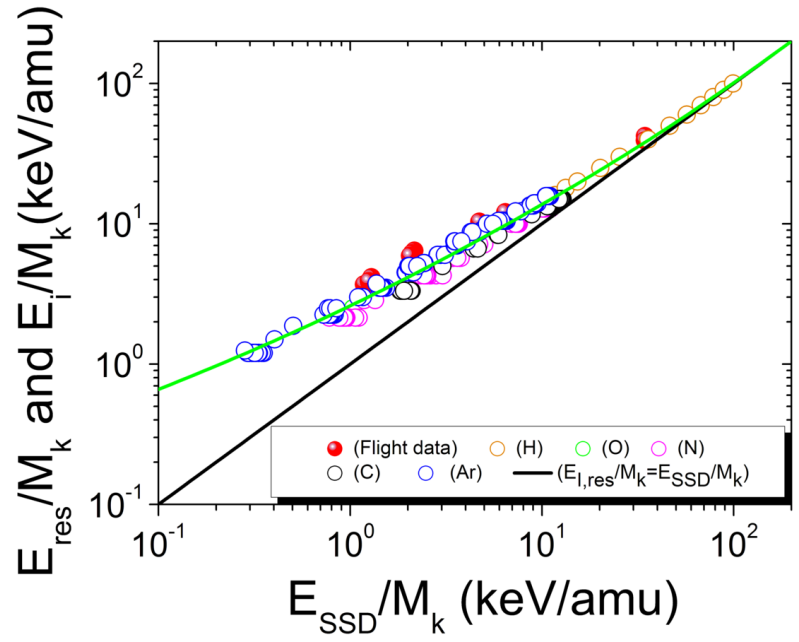

Fig. 13. Comparison of the flight calibration data of the energy before hitting the SSD versus the mean measured energy with the SSDs. The data are from STEREO-A/PLASTIC as in Fig. 12 (red full data points). All other symbols and colours correspond to calibration results for different species and diverse energies. The green curve represents our model for energy ranges up to $200 \mathrm{keV} / \mathrm{amu}$.

straight diagonal line in Fig. 11. In this case, we will measure all the incident energy (from different energy stages for different ions).

Thus, our model for the response of our SSD mean measured energy is based on the following expression:

$E_{i}=A M_{k}\left(\frac{E_{\mathrm{SSD}}}{M_{k}}\right)^{B} \exp \left(-C \frac{E_{\mathrm{SSD}}}{M_{k}}\right)+E_{\mathrm{SSD}}$

or

$E_{i}=A_{m}\left(E_{\mathrm{SSD}}\right)^{B_{m}} \exp \left(-C_{m} E_{\mathrm{SSD}}\right)+E_{\mathrm{SSD}}$

respectively.

The parameters $A, B$, and $C$ were first derived from the direct calibration of the SSD and the IFM1. They were then derived from the recalibration of the flight data obtained by STEREO-A (see Figs.12 and 13).

The residual energy $\left(E_{\text {res }}\right)$ was calculated according to Fig. 12 for each element using the following expression (valid for the SSD-side with a path length $l$ of $0.08 \mathrm{~m}$ ):

$\frac{E_{\text {res }}}{M_{k}}=\frac{1}{3 \cdot 10^{-5}(\mathrm{TOF}[\mathrm{ns}])^{2}} \mathrm{keV} / \mathrm{amu}$

The bulk solar wind velocity range in our Figs. 12 and 13 was between 250 and $700 \mathrm{~km} / \mathrm{s}$, and both, slow and fast solar wind regimes, were included.

In Fig. 14, we see how our parameters $A_{m}, B_{m}$, and $C_{m}$ depend on the atomic masses of the elements considered.

$A_{m}$ has a power law dependence on the mass. $B_{m}$ is a constant, with a value of $0.47+/-0.03$, which compares well with the value from stopping power theory of $0.5 . C_{m}$ is inversely proportional to the mass. 


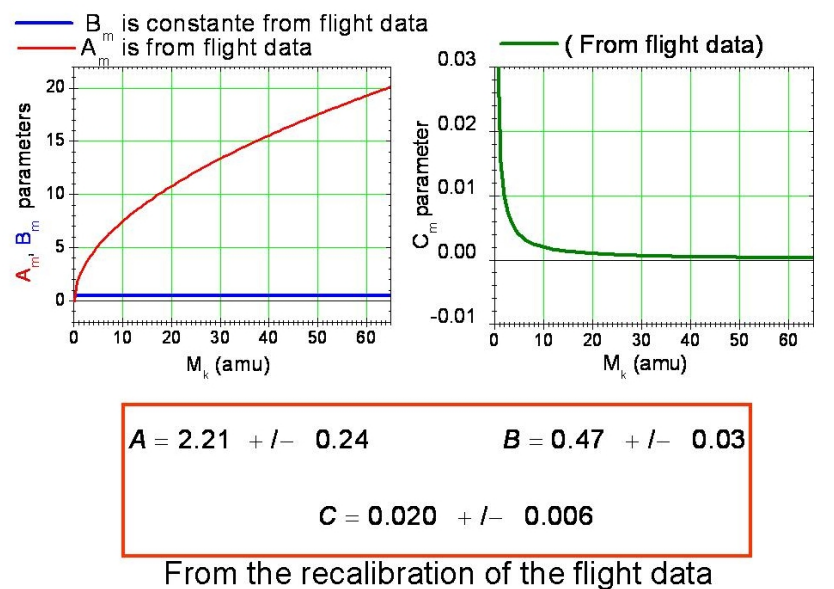

Fig. 14. Dependency of the parameters $A_{m}$ (red curve), $B_{m}$ (blue line), and $C_{m}$ (olive curve) as functions of the atomic masses of the elements. The parameters are derived from flight data.

Generally, for $\mathrm{H}$ and $\mathrm{He}$ up to energies of $250 \mathrm{keV}$ and $800 \mathrm{keV}$ respectively, we will be able to measure the incident energy with the SSD. However, it is impossible to cover the full range in the cases of $\mathrm{O}$ and $\mathrm{Fe}$ (see Fig. 15).

Using the same condition parameters as in Fig. 5a, and using our model of the TOF and SSD energy response, and the different conversions from channels to ns and to $\mathrm{keV}$, we can calculate the predicted TOF and $E_{S S D}$. A result is plotted in a $\left(E_{\mathrm{SSD}}, \mathrm{TOF}\right)$ contour plot of the flight data from 22 January $2007\left(V_{a}=17.3 \mathrm{kV}\right)$ as measured by STEREO-A (Fig. 16). In this figure, the diamond symbols show the predicted location of the peaks for various oxygen and iron charge states.

The simulations of the charge state distributions of oxygen and iron in this study were based on an application of recombination and ionization rates in static equilibrium from (Arnaud and Rothenflug, 1985; Arnaud and Raymond, 1992), using an electron temperature for the corona of about $1.6 \cdot 10^{6} \mathrm{~K}$.

\subsection{Deriving the mass and charge state distribution with correct physical units from flight data}

In order to derive the correct mass and charge state of the species entering the PLASTIC sensor, we are presenting a new method, which is based on stopping power theory (Sect. 3.2) and on the SSD energy response (Sect. 3.3). With the measured parameters $\left(E / q_{k}\right.$, TOF, $\left.E_{\mathrm{SSD}}\right)$ for each measured event and for a given $V_{a}$, we can solve the following non-linear system of equations (NLSQ). $E_{\text {tot }}$ represents the total energy of an ion before crossing the carbon foil and after

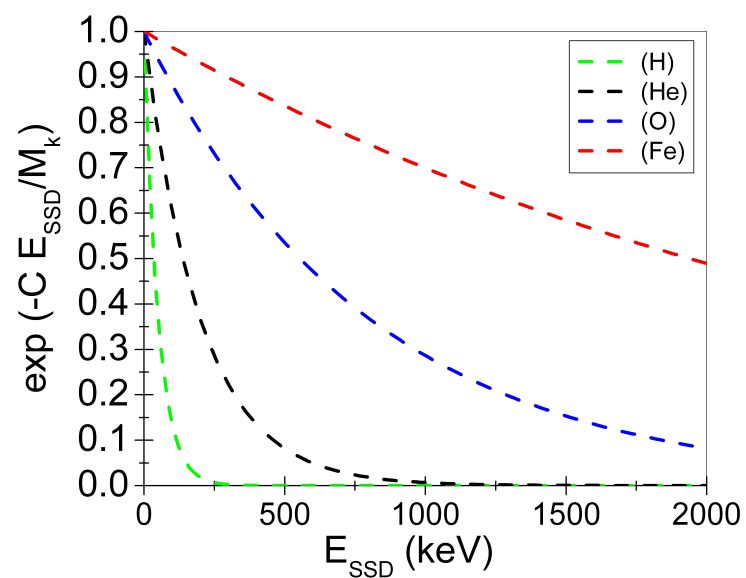

Fig. 15. Simulated effect of the exponential decrease of $C_{m}$ for $\mathrm{H}$, $\mathrm{He}, \mathrm{O}$ and $\mathrm{Fe}$.

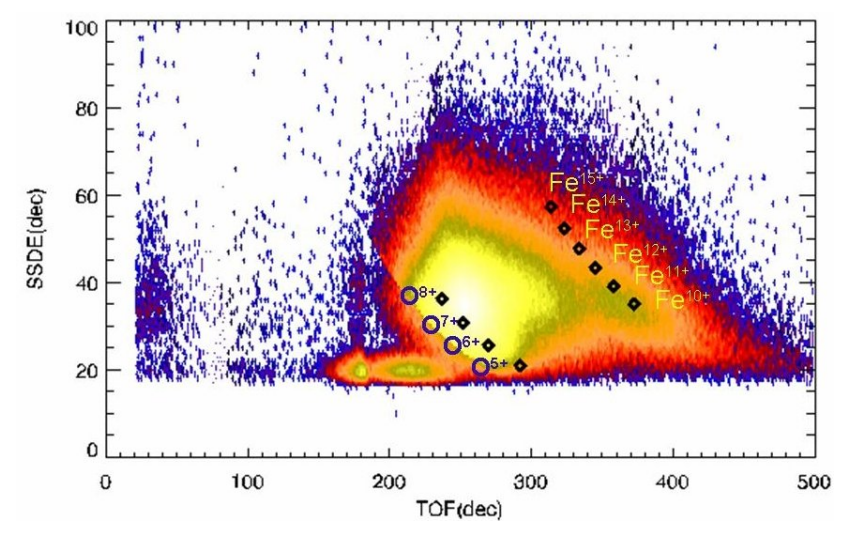

Fig. 16. Contour plot of the energy measured by the SSD versus the TOF measured (flight data 22 January 2007). Counts are accumulated over a whole day, for all quadrants, all sections and all priorities and were measured by the PLASTIC instrument on STEREOA. The mean bulk solar wind velocity is $440 \mathrm{~km} / \mathrm{s}$. Diamonds show the expected location of the peaks of our TOF and $E_{\mathrm{SSD}}$ for $\mathrm{O}$ and Fe with different charge states.

the acceleration zone:

$$
\begin{aligned}
M_{k}= & 2\left(\frac{\mathrm{TOF}}{l}\right)^{2}\left[A M_{k}^{1-B} E_{\mathrm{SSD}}^{B} \exp \left(-C \frac{E_{\mathrm{SSD}}}{M_{k}}\right)+E_{\mathrm{SSD}}\right] \\
Z_{k}= & f\left(M_{k}\right) \\
E_{\mathrm{tot}} & =\frac{M_{k}}{2\left(\frac{\mathrm{TOF}}{l}\right)^{2}}+d \frac{231.6 \rho Z_{\mathrm{keff}}^{7 / 6} Z_{2} \sqrt{E_{\mathrm{tot}}}}{\left(Z_{\mathrm{keff}}^{2 / 3}+Z_{2}^{2 / 3}\right)^{3 / 2} M_{2} \sqrt{M_{k}}} \\
& +d \frac{509.59 \rho Z_{k} Z_{2} M_{k}}{M_{2}\left(M_{k}+M_{2}\right)\left(Z_{k}^{0.23}+Z_{2}^{0.23}\right)} S_{n}(\varepsilon)
\end{aligned}
$$

The relation between the atomic number and the mass was included in the algorithm as a data matrix that contains information about the properties of the most abundant isotopes from $\mathrm{H}$ to $\mathrm{Cu}$. 


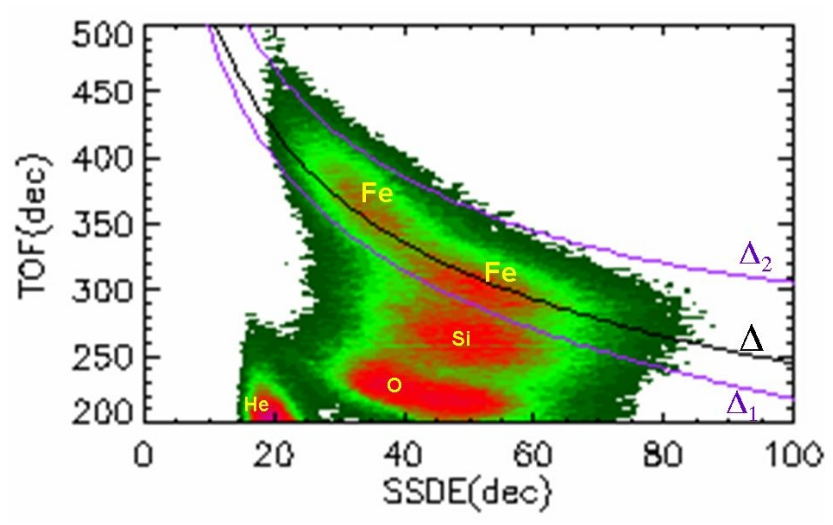

Fig. 17. Contour plot of PHA data of an accumulation of DOY 120 (2007) and DOY 126 (2007), which correspond respectively to FSW $(700 \mathrm{~km} / \mathrm{s})$ and SSW $(280 \mathrm{~km} / \mathrm{s})$. The TOF versus the energy measured with a SSD is shown; Fe, O, Si and some He data are observed. The data are from STEREO-A/PLASTIC.

The NLSQ can be solved through the use of a comprehensive reverse communication routine C05NDF from the NAG library. The routine is based on a modification of the Powell hybrid method (Moré et al., 1980). It chooses the correction at each step as a convex combination of the Newtonian and scaled gradient directions. The starting points for $E_{\text {tot }}$ were the SSD measured energy $\left(E_{\mathrm{SSD}}\right)$, while the masses used were virtual ones calculated directly from the TOF and $E_{\text {SSD }}$ raw events without corrections. The atomic numbers were calculated by taking the image of the virtual mass (without any correction) using a simple fit function derived from the periodic table data. The program converges without any problems, and the solution is given in the form of $\left(M_{k}, Z_{k}\right.$, $\left.E_{\text {tot }}\right)$ for each event. The corresponding charge event can be calculated using the following formula:

$q_{k}=\frac{E_{\mathrm{tot}}}{\left(\frac{E}{q_{k}}+V_{a}\right)}$

We checked the solutions of this algorithm by using the IFM1 calibration PHA data sets for different light, medium and heavy elements such as $\mathrm{H}, \mathrm{He}, \mathrm{O}$ and $\mathrm{Ar}$, as well as for various charge states. This yielded satisfactory results.

We also ran tests with flight data, and the result of the mass for oxygen, for example, without corrections was around $8 \mathrm{amu}$. However, with our algorithm, the corrected mass was $16 \pm 1.5 \mathrm{amu}$. For Fe, the non-corrected masses derived directly from the measurements was around $20 \mathrm{amu}$, while, with our algorithm, the results was $56 \pm 7.5 \mathrm{amu}$. The errors are the Half Full Width at Half Maximum (HFWHM).

\section{Data filtration}

The STEREO data are made available from the Mission Operation Control (MOC); they are transmitted to the Univer- sity of New Hampshire as "level 0", where they are converted into "level 1" data and distributed in Common Data Format (CDF). The CDF contains a collection of different ASCIIs files, such as the PHA, the Full Monitor Rate (FMR), and the Normal Monitor Rate (NMR). Only a maximum selection of 768 events, from all quadrants and channels, for each of the 128 complete $E / q_{K}$ cycle and 32 defection angle steps are registered in the PHA in full resolution. We were interested mainly in a minor solar wind ions and particularly in Fe ions. Minor ions are good tracers for turbulent processes and interact efficiently with fluctuations on scales directly involved in the heating process of the solar wind (Zurbuchen, 1996). Minor ions have a higher priority in the PHA files, and only such files contain sufficient information for each measured event in the form of:

1. Sweeping ESA step (SWPE ranging from step $j=0$ to $j=127$ )

2. Deflection step (SWPD ranging from step 0 to 31 )

3. Energy measured by the SSD (ssde from channel 0 to 1023)

4. The position in the SSD pixel (ssd_id ranging from pixel 0 to 15$)$

5. The time of flight (TOF from channel 0 to 1023)

The flight measurements are registered in the following way:

- For different quadrants $\left(\mathrm{Q}_{0}, \mathrm{Q}_{1}, \mathrm{Q}_{2}, \mathrm{Q}_{3}\right)$

- For different sections $(0,1,2,3)$

- For different priorities $(0,1,2,3)$ etc.

On the basis of the SSD model of the mean measured energy for non channeled ions, we can express the TOF as a function of the energy measured by the SSD (ssde). The corresponding Fe curve for the mean measured energy is shown as $\Delta$ in a Fig. 17. The modus operandi is a universal criterion, which consists in extracting all $\mathrm{Fe}$ data that are between the two violet curves $\Delta_{1}$ and $\Delta_{2}$, see Fig. 17 and Daoudi (2008).

\section{Conclusions}

The research covered by this paper includes the analytical modeling of the response of the ESEA, the TOF, and of the SSDs of the PLASTIC instrument. The result is a new data reduction algorithm which is simple to implement and which will be of use in the analysis of data sets of a similar nature. We tested the algorithm using the raw flight data and found that it works well, allowing us to obtain the mass and the charge state corrected for energy defects in the carbon foil and in solid-state detectors. Finally, in order to get a better mass and charge state resolution, the flight data measurements were filtered. The modus operandi to extract the $\mathrm{Fe}$ data with the above-mentioned algorithm can provide charge 
states with a high time resolution of 5 min or less. As the method is sensitive for every measured event, it can become useful in research on the solar wind and solar processes by studying the elemental and isotopic composition of the solar wind ions at $1 \mathrm{AU}$, as well as the charge state distribution of minor ions. PLASTIC will allow the study of the origin of the SSW, and the transitions from SSW to FSW, and from FSW to SSW during the early phase of the STEREO mission, which is during the solar activity minimum. That will enable us to draw new conclusions for the SSW and FSW as well as the transient phenomena (Galvin et al., 2008). It can become also useful in future research on Interplanetary Coronal Mass Ejections (ICMEs) and their boundaries by measuring in situ the ICME properties as they pass the STEREO spacecraft. PLASTIC data will enable us to study the ionization state of the solar wind ions. Elevated charge states of elements such as iron are often observed in ICMEs, as well as unusual elemental compositions. Measuring the frozen-in ionic charge states of the solar wind plasma with high time resolution will allow the study of the CME initiation process on the Sun. PLASTIC will also help to obtain more information on the solar atmosphere prior to CME initiation via the study of the elemental composition (Galvin et al., 2008).

Acknowledgements. This work is supported by the Swiss National Foundation.

Edited by: B. Heber

Reviewed by: two anonymous referees

\section{References}

Aellig, M. R.: Freeze-in Temperatures and Relative Abundances of Iron Ions in the Solar Wind Measured with SOHO/CELIAS/CTOF, Ph.D. Thesis, University of Bern, Switzerland, 133, 1998.

Allegrini, F., Bochsler, P., Wimmer-Schweingruber, R. F., and Wurz, P.: Determination of Low-Energy Ion Induced Electron Yields from Thin Carbon Foils, Nucl. Instrum. Meth. B., 211, 487-494, 2003.

Arnaud, M. and Rothenflug, R.: An Update evaluation of recombination and ionization rates, Astron. Astrophys., Suppl., 60, 425457, 1985.

Arnaud, M. and Raymond, J.: Iron ionization and recombination rates and ionization equilibrium, Astrophys. J., 398, 394-406, 1992.

Betz, G. and Wien, K .: Energy and angular distributions of sputtered particles, Int. J. Mass. Spectr, 140, 1-110, 1994.

Biersack, J. P.: The Calculation of Ion Ranges in Solids with Analytical Solutions, Springer series on electrophysics, 10, 157-166, 1982.

Blush, L. M., Bochsler, P., Daoudi, H., Galvin, A., Karrer, R., Kistler, L., Klecker, B., Möbius, E., Opitz, A., Popecki, M., Thompson, B., Wimmer-Schweingruber, R. F., and Wurz, P.:
Development and calibration of major components for the STEREO/ PLASTIC (plasma and suprathermal ion composition) Instrument, Adv. Space Res., 36, 1544-1556, 2005.

Bochsler, P.: Abundances and charge states of particles in the solar wind, Reviews of Geophysics, 38, 247-266, 2000.

Daoudi, H.: First STEREO/PLASTIC measurements of Fe charge states in the solar wind; presentation of a new method for flight data analyses, PhD. Thesis, Univ. of Bern, Switzerland, 176, 2008.

Galvin, A. B., Kistler, L. M., Popecki, M. A., Farrugia, C. J., Simunac, K. D. C., Ellis, L., Möbius, E., Lee, M. A., Boehm, M., Carroll, J., Crawshaw, A., Conti, M., Demaine, P., Ellis, S., Gaidos, J. A., Googins, J., Granoff, M., Gustafson, A., Heirtzler, D., King, B., Knauss, U., Lavasseur, J., Longworth, S., Singer, K., Turco, S., Vachon, P., Vosbury, M., Widholm, M., Blush, L. M., Karrer, R., Bochsler, P., Daoudi, H., Etter, A., Fischer, J., Jost, J., Opitz, A., Sigrist, M., Wurz, P., Klecker, B., Wimmer-Schweingruber, R. F., Koeten, M., Thompson, B., and Steinfeld, D.: The Plasma and Suprathermal Ion Composition (PLASTIC) Investigation on the STEREO Observatories, Space Science Rev., 136, 437-486, doi:10.1007/s11214-007-9296-x, 2008.

Hefti, S.: Solar Wind Freeze-in Temperatures and Fluxes Measured with SOHO/ CELIAS/CTOF and calibration of the CELIAS Sensors, Ph.D. Thesis, Univ. Bern, Switzerland, 103, 1997.

Hohl, M.: MEFISTO II, Design, setup, characterization and operation of an improved calibration facility for solar plasma instrumentation, Ph.D. Thesis, Univ. Bern, Switzerland, 131, 2002.

Karrer, R.: Ion optical calibration of the PLASTIC sensor on STEREO, Ph.D. Thesis, Univ. Bern, Switzerland, 207, 2007.

Klein, K. M., Park, C., and Tasch, A. F.: Monte Carlo simulation of boron implantation into single-crystal silicon, Electron Devices, IEEE Transactions on, 39, 1614-1621, 1992.

Marti, A., Schletti, R., and Wurz, P., and Bochsler, P.: Calibration facility for solar wind plasma instrumentation, Review of scient. instr., 72, 1354-1360, 2000.

Moré, J. J., Garbow, B. S., and Hillstrom, K. E.: User guide for MINPACK-1, Argonne Nat. Lab., Techn. report ANL, 80-74, 1980.

Oetliker, M.: CTOF, A Solar Wind Time of Flight Mass Spectrometer with High Charge Resolution (Numerical simulations and Calibrations), Ph.D. Thesis, Univ. Bern, Switzerland, 133, 1993.

Pilz, W., Borany, J. V., Grötzschel, R., Jiang, W., Posselt, M., and Schmidt, B.: Dependence of the silicon detector response to heavy ions on the direction of incidence (Computer simulations versus experimental data), Nucl. Instrum. Meth. A: 419, 137145, 1998.

Wimmer-Schweingruber, R. F.: Solar and galactic composition, AIP Conference Proceedings Subseries on Astronomy and Astrophysics, 598, SOHO/ACE Workshop, Bern, Switzerland, 69 March, 3-9, 2001.

Ziegler, J. F., Biersack, J. P., and Littmark, U.: The stopping and range of Ions in solids, Pergamon press, Inc., 1, Maxwell House, Fairview Park, Elmsford, New York 10523, U.S.A, 321, 1992.

Zurbuchen, T.: Turbulence in the Interplanetary Medium and its Implications on the Dynamics of Minor Ions. Ph.D. Thesis, Univ. Bern, Switzerland, 138, 1996. 